\title{
ESTUDIO IN VITRO DE LA DEFICIENCIA DE ACIL-COA DESHIDROGENASA DE CADENA MUY LARGA CON AFECTACION MUSCULAR
}

José Henry Osorio ${ }^{1}$

\section{RESUMEN}

Introducción: Los estudios in vitro, que buscan intermediarios de la degradación mitocondrial de ácidos grasos, facilitan el diagnóstico de alteraciones hereditarias o adquiridas en esa ruta metabólica. Objetivo: Analizar la producción de metabolitos en fibroblastos de pacientes con deficiencia de acil-CoA deshidrogenasa de cadena muy larga (VLCAD) con afectación muscular, incubados con ácido oleico deuterado. Materiales y Métodos: Se incubaron fibroblastos de tres pacientes con deficiencia de acil-CoA deshidrogenasa de cadena muy larga (VLCAD) con cardiomiopatía y de 10 controles en presencia de ácido oleico deuterado. Resultados: Se encontró un perfil característico luego de la incubación de fibroblastos con esta deficiencia. Conclusión: Este sustrato podría ser usado para realizar diagnóstico in vitro de la deficiencia de VLCAD con afectación muscular.

Palabras clave: ácidos grasos, metabolismo, $\beta$-oxidación mitocondrial.

\section{IN VITRO STUDY OF VERY LONG CHAIN ACYL-COA DEHYDROGENASE DEFICIENCY WITH MUSCULAR INVOLVEMENT}

\section{ABSTRACT}

Introduction: In vitro studies for searching intermediates of mitochondrial fatty acid degradation, are a tool for diagnosis of hereditary or adquire alterations of the above mentioned metabolic pathway. Objective: To analize the metabolite production in fibroblasts from patientes with very long chain acylCoA deficiency and muscular involvement incubated with deuterated oleic acid. Materials and Methods: Fibroblasts of three patients with very long chain acyl-CoA deficiency and cardiomyopathy and ten control were incubated with deuterated oleic acid. Results: It was found a characteristic profile after incubation of fibroblats with this deficiency. Conclusion: This substrate could be used to perform in vitro diagnosis of VLCAD deficiency with musclar involvement.

Key words: fatty acids, metabolism, mitochondrial $\beta$-oxidación.

\footnotetext{
${ }^{1}$ pH D, Departamento de Ciencias Básicas de la Salud, Universidad de Caldas. Manizales, Colombia. Correo electrónico: jose.osorio_o@ucaldas.edu.co
} 


\section{INTRODUCCIÓN}

El diagnóstico de la deficiencia de VLCAD, además de la clínica, involucra otros hallazgos, como una baja actividad enzimática de la misma. Los análisis bioquímicos en estos pacientes informan niveles elevados de C14n9 en el análisis de ácidos grasos libres en plasma, por cromatografía de gases; niveles elevados de ácidos dicarboxílicos desde C6 hasta C14 en el análisis de ácidos orgánicos en orina, por cromatografía de gases acoplada a espectrometría de masas; y niveles altos de las acilcarnitinas C14:1, C14 y C16 en el análisis de sangre por espectrometría de masas en tándem (1). Las acilcarnitinas producidas luego de la oxidación mitocondrial de ácidos grasos, pueden salir de la mitocondria y acumularse en el medio de cultivo, mientras que los tioésteres acil-CoA formados, quedan confinados en la mitocondria debido a su incapacidad para atravesar la membrana mitocondrial (2). A partir de ese precepto, Nada et al. (3) diseñaron un método para el diagnóstico de las deficiencias de la B-oxidación mitocondrial de los ácidos grasos, mediante la incubación de fibroblastos utilizando ácido [17,17,18,18-2H4]-9,12-octadecadienoico, (ácido linoleico deuterado), en presencia de L-carnitina; utilizando espectrómetrometría de masas en tándem, para medir las acilcarnitinas deuteradas en el medio de cultivo. Mediante ese procedimiento, se consigue establecer el diagnóstico de varias deficiencias de la B-oxidación mitocondrial, pero a pesar de ser una técnica altamente sensible y precisa, tiene el inconveniente de la accesibilidad para numerosos laboratorios en el mundo, dado los costos que implica, por lo que se han diseñado otras técnicas que determinan acilcarnitinas en medios de cultivo de fibroblastos por cromatografía de gases acoplada a espectrometría de masas con monitoreo selectivo de un ion (GC-CI-MS y GC-MS) (4). En este trabajo se presentan los resultados de la incubación de fibroblastos con ácido oleico deuterado, detectando metabolitos por cromatografía de gases acoplada a espectrometría de masas, en pacientes que sufren VLCAD caracterizada por afectación muscular. El presente estudio analizó la producción de intermediarios por incubación de fibroblastos con ácido oleico deuterado, como herramienta diagnóstica en pacientes con deficiencia de VLCAD.

\section{MATERIALES Y MÉTODOS}

El presente estudio es de tipo experimental. El material biológico empleado fue 10 cultivos diferentes de fibroblastos normales y de tres pacientes con deficiencia de VLCAD que presentaban episodios de miopatía con rabdomiólisis. Como sustrato fue empleado el ácido oleico deuterado (Cambridge Isotope Laboratories). Fueron utilizados los siguientes compuestos deuterados para la preparación de la curva de calibración: [8,8,8-d3]octanoil-Lcarnitina.HCl, [10,10,10-d3]decanoil-L-carnitina. $\mathrm{HCl},[12,12,12-\mathrm{d} 3]$ dodecanoil-L-carnitina. $\mathrm{HCl},[14,14,14-\mathrm{d} 3]$ tetradecanoil-L-carnitina. $\mathrm{HCl},[16,16,16-\mathrm{d} 3]$ hexadecanoil-L-carnitina. $\mathrm{HCl}$ (Ten Brinx-Free University Amsterdam). Como estándar interno fue adicionado ácido undecanodioico (Fluka), durante el proceso de extracción de las muestras.

Cultivo de fibroblastos. Los fibroblastos (420 pasajes) fueron cultivados en medio de cultivo similar al medio basal Eagle's con bicarbonato y HEPES [4-(2-hidroxietil)-1-ácido piperazinaetano sulfúrico], (MEM: medio mínimo esencial), suplementado con suero de bovino recién nacido $10 \%(\mathrm{v} / \mathrm{v})$ y gentamicina $1 \%(\mathrm{v} / \mathrm{v})$ a $37^{\circ} \mathrm{C}$, en estufa con $5 \% \mathrm{CO}_{2} / 95 \%$ de aire. Después de alcanzar el punto de confluencia (80-100\%), las células fueron lavadas dos veces con PBS (buffer fosfato salino), la solución se tripsinizó (1 $\mathrm{ml}$ de tripsina-EDTA a $37^{\circ} \mathrm{C}$, y posteriormente se neutralizó mediante la adición de 3-5 $\mathrm{ml}$ de MEM. Las células fueron transferidas y centrifugadas a $337 \mathrm{X} \mathrm{g}(5 \mathrm{~min}$, $\left.20^{\circ} \mathrm{C}\right)$ en tubos cónicos de $10 \mathrm{ml}(0,8-1,2 \mathrm{mg}$ proteína), quedando listas para ser incubadas en presencia de sustratos deuterados. Se utilizó el método de Lowry et al. (5) para la determinación de proteínas. 
Oxidación de ácido oleico deuterado en fibroblastos. Para la oxidación de ácido oleico deuterado en fibroblastos fue utilizada la técnica diseñada por Osorio (6); se preparó una solución de medio de cultivo con una concentración final de 0,15 mM de ácido ácido oleico deuterado, 1 mM BSA, y L-carnitina 0,2 mM. La incubación por los fibroblastos se llevó a cabo de la siguiente manera: después de la tripsinización, las células fueron resuspendidas en MEM enriquecido en frascos falcon de $2,5 \mathrm{ml}$ (2 frascos por caso). Después de 72 h de incubación, el medio de cultivo fue recogido mediante centrifugación y almacenado a $-20^{\circ} \mathrm{C}$ hasta su análisis. Las células se resuspendieron en $1 \mathrm{ml}$ de PBS1 para proceder a la determinación de proteínas. El análisis cuantitativo se basó en el método del estándar interno. Se prepararon curvas de calibración para los ácidos grasos C8, C10, C12 y C14, en un rango de 0 a $150 \mathrm{nM}$, y C16 en un rango de 0-1000 $\mathrm{nM}$, utilizando acilcarnitinas deuteradas en el último carbono, diluidas en MEM ( $p<0,00001$ para cada curva de calibración). El análisis de los ácidos grasos de los medios enriquecidos (curvas de calibración y productos de la incubación) fue realizado después de la hidrólisis con $\mathrm{KOH}$. Las curvas de calibración se obtuvieron mediante el análisis de regresión lineal, representando la relación de concentraciones de cada ácido respecto al estándar interno frente a la relación de áreas.

Condiciones de la cromatografía gas-líquido. Flujo de gas portador (He): $1 \mathrm{ml} / \mathrm{min}$; división de flujo: 1:30; tiempo de inyección: $1 \min 5$ segundos; temperatura del inyector: $250^{\circ} \mathrm{C}$; temperatura del horno y programación de temperaturas: $\mathrm{T} 170^{\circ} \mathrm{C}$ a $6^{\circ} \mathrm{C} / \mathrm{min}, \mathrm{T} 2250^{\circ} \mathrm{C}$ a $20^{\circ} \mathrm{C} / \mathrm{min}$ hasta $\mathrm{T} 3300^{\circ} \mathrm{C}$; volumen de inyección: $1 \mathrm{ml}$; tiempo total: $38,5 \mathrm{~min}$. Las condiciones de la espectrometría de masas fueron las siguientes: el análisis cualitativo se realizó por cromatografía de gases-espectrometría de masas, con impacto electrónico y monitorización selectiva de iones. Para cada ácido se monitorizó el ion M-15, ya que es el ion selectivo y uno de los más abundantes; la ionización se realizó por impacto electrónico a $70 \mathrm{eV}$ y la fuente de iones se mantuvo a una temperatura de $200^{\circ} \mathrm{C}$. La temperatura del analizador fue de $100^{\circ} \mathrm{C}$; para la adquisición de datos, el instrumento utilizó un scanning repetitivo en un rango de 40 a 600 unidades de masa atómica. Fue utilizado un cromatógrafo de gases 5890 Series II Plus (Espectrómetro de masas Hewlett Packard serie 5972, Palo Alto, CA, año 2000).

El manejo de datos se hizo utilizando Microsoft Excel y Minitab Statistical Software.

De acuerdo con el artículo 11 literal a. de la Resolución No. 8430 de 1993 del Ministerio de Salud, de Normas Científicas, Técnicas y Administrativas para la Investigación en Salud, el presente estudio es considerado sin riesgo. Los pacientes firmaron consentimiento informado y el trabajo fue aprobado por el respectivo Comité de Ética.

El autor declara no tener conflicto de intereses alguno.

\section{RESULTADOS}

En todas las curvas de calibración se obtuvo un coeficiente de correlación superior al 0,99. La Figura 1 muestra el cromatograma GC-MS-SIM de ácidos grasos en fibroblastos incubados con ácido 2-oleico deuterado. Los ácidos grasos saturados no deuterados corresponden a la oxidación del ácido palmítico del medio de cultivo. Se observaron solamente los metabolitos deuterados de 12, 14 y 16 átomos de carbono (D2C12:1), (D2C14:1), (D2C16:1), respectivamente, producto de la degradación del ácido oleico deuterado. La Tabla 1 muestra las concentraciones observadas para los diferentes ácidos grasos deuterados. Con el análisis estadístico mediante la prueba $t$ de Student se encontró diferencia significativa $(p<0,05)$ entre los datos de los pacientes y los de los controles, para los ácidos grasos deuterados de 14 y 16 átomos de carbono. 
Estudio In vitro de la deficiencia de Acil-CoA deshidrogenasa de cadena muy larga con afectacion muscular

Tabla 1. Producción de ácidos grasos deuterados en fibroblastos incubados con ácido oleico deuterado.

\begin{tabular}{ccccc}
\hline $\begin{array}{c}\text { ÁCIDOS GRASOS } \\
\text { INTERMEDIARIOS }\end{array}$ & PROMEDIO & INTERVALO & PROMEDIO & p \\
(nmol/mg proteína/72 h) & CONTROLES & CONTROLES & VLCAD-M & \\
& & & & \\
\hline D2C12:1 & 3,6 & $2,8-4,2$ & 2,9 & - \\
D2C14:1 & 2,9 & $1,9-5,1$ & 20,1 & 0,0234 \\
D2C16:1 & 3,2 & $2,5-3,8$ & 10,9 & 0,0148 \\
\hline
\end{tabular}

Abreviaturas: Ácidos grasos deuterados de 12 átomos de carbono (D2C12:1) dodecenoico deuterado; de 14 átomos de carbono (D2C14:1) tetradecenoico duterado; de 16 átomos de carbono (D2C16:1) hexadecenoico deuterado, producto de la degradación del ácido oleico deuterado.
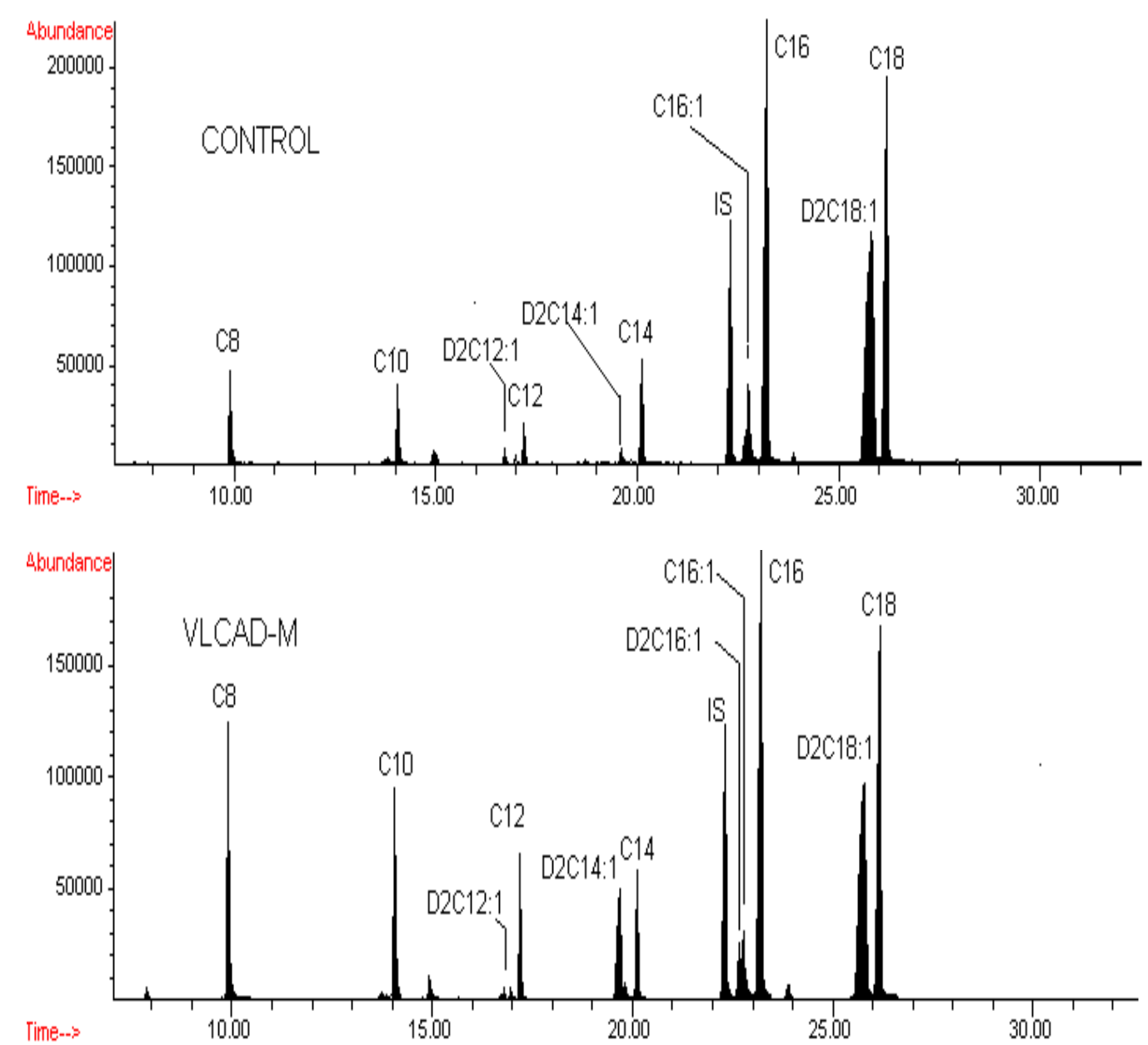

Están indicados los ácidos grasos del medio de cultivo, según el número de átomos de carbono. C8: octanoico; C10: decanoico; C12:1: dodecenoico; C12: dodecanoico; C14:1: tetradecenoico; C14: tetradecanoico; C16: hexadecanoico; $\mathrm{OH}-\mathrm{C} 16: 0$ : hidroxihexadecanoico; así como los ácidos grasos deuterados, de 12 átomos de carbono (D2C12:1) dodecenoico; 14 átomos de carbono (D2C14:1) tetradecenoico deuterado; y 16 átomos de carbono (D2C16:1) hexadecenoico deuterado, producto de la degradación del ácido oleico deuterado. IS identifica el estándar interno.

Figura 1. GC-MS-SIM de la incubación del ácido 2-oleico deuterado en fibroblastos control y de pacientes con deficiencia de VLCAD-M. 


\section{DISCUSIÓN}

La enzima acil-CoA deshidrogenasa de cadena muy larga (VLCAD) es un homodímero de 154-Kda (7) y su cADN consta de una región de 2.177 bp codificando un péptido líder de 40 aminoácidos y un polipéptido maduro de 615 aminoácidos, para un total de 655 aminoácidos en la proteína entera (8). Esta enzima es específica para acil-CoAs de 14-24 átomos de carbono y cataliza más del $90 \%$ de la deshidrogenación del palmitoil-CoA $(\mathrm{C} 16)(9,10)$. El gen se encuentra localizado en el cromosoma 17p13 (11) y esta enzima es considerada un paso limitante en la oxidación de ácidos grasos de cadena larga (12). Con base en la clínica presentada en esta deficiencia se han informado 3 fenotipos: una forma infantil severa (10), una forma de presentación más benigna que se presentan también en la niñez (13) y una forma muscular, la cual se manifiesta después de la niñez (14). Más recientemente estas variantes han sido descritas respectivamente como VLCAD-C por presentar cardiomiopatía dilatada, VLCAD-H caracterizada por episodios de hipoglicemia, y VLCAD-M en la cual se presentan episodios de miopatía con rabdomiólisis (15). La detección de metabolitos intermediarios de la $\beta$-oxidación mitocondrial de los ácidos grasos, ha sido ampliamente estudiada (16-18). La espectrometría de masas en tándem sigue siendo el método más efectivo para detectar alteraciones en esta vía metabólica (19). Sin embargo, es una técnica que no está disponible para muchos laboratorios, por lo cual se deben buscar otras alternativas. El uso de fibroblastos para el análisis del funcionamiento de la $\beta$-oxidación mitocondrial de los ácidos grasos, mediante la incubación con diferentes sustratos ha sido plenamente documentada (20-22). La técnica de análisis de ácidos grasos mediante GC/MS ha probado ser efectiva para este tipo de análisis; en trabajos anteriores, trabajando con ácido palmítico deuterado, han sido encontrados elevados los niveles de diferentes ácidos grasos, después de hidrolizar las acilcarnitinas presentes en el medio de cultivo de los fibroblastos de los pacientes que presentaban deficiencias de la $\beta$-oxidación mitocondrial de los ácidos grasos (6). En el presente estudio ha sido encontrado un perfil que muestra incremento en los niveles de varios ácidos grasos deuterados luego de realizar incubación de fibroblastos de pacientes con deficiencia de VLCAD-M con ácido oleico deuterado, principalmente, metabolitos de 14 (D2C14:1) y 16 (D2C16:1) átomos de carbono. Estos resultados refuerzan el diagnóstico, toda vez que en otros estudios in vitro de esta deficiencia, al ser incubados con ácido palmítico deuterado, también se ha encontrado alterado el perfil metabólico (23). La principal ventaja de este método, es la posibilidad de obviar el uso de espectrometría de masas en tándem, dada la dificultad para implementar esta técnica por costos, en algunos laboratorios. La metodología es similar a la utilizada para realizar incubaciones con ácido palmítico deuterado, sin embargo, el uso del ácido oleico deuterado facilita la discriminación entre los diferentes tipos de deficiencia de VLCAD, mostrando los ácidos grasos insaturados que se acumulan en cada tipo de deficiencia, caso que no es posible cuando se incuban los fibroblastos con un ácido graso saturado deuterado como el palmítico. 


\section{REFERENCIAS}

1. Merritt JL 2nd, Vedal S, Abdenur JE, Au SM, Barshop BA, Feuchtbaum L, et al. Infants suspected to have very-long chain acyl-CoA dehydrogenase deficiency from newborn screening. Mol Genet Metab $2014 ; 111(4): 484-92$.

2. Kler RS, Jackson S, Bartlett K, Bindoff LA, Eaton S, Pourfarzam M, et al. Quantitation of acyl-CoA and acylcarnitine esters accumulated during abnormal mitochondrial fatty acid oxidation. J Biol Chem $1991 ; 266: 22932-38$.

3. Nada MA, Chace $D H$, Sprecher $H$, Roe CR. Investigation of B-oxidation intermediates in normal and MCAD deficient human fibroblasts using tandem mass spectrometry. Biochem Mol Med 1995; 54:5966.

4. Ventura FV, Costa CG, Struys EA, Ruiter J, Allers P, Ijlst L, et al. Quantitative acylcarnitine profile in fibroblasts using U-13Cpalmitic acid: an improved tool for the diagnosis of fatty acid oxidation defects. Clin Chim Acta 1999; 281:1-17.

5. Lowry OH, Rosebrough NJ, Farr AL, Randall RJ. Protein measurement with the Folin phenol reagent. J Biol Chem 1951; 193:265-75.

6. Osorio JH. Producción de metabolitos en fibroblastos incubados con ácido oleico deuterado. Archiv Med (Manizales) 2013; 13(2):202-207.

7. Aoyama T, Ueno I, Hashimoto T. Rat very-long-chain acyl-CoA dehydrogenase, a novel mitochondrial acyl-CoA dehydrogenase gen product, is a rate-limiting enzyme in long-chain fatty acid $\beta$-oxidation system. The cDNA and deduced amino acid sequence and distinct specificities of the cDNA-expressed protein. J Biol Chem 1994; 269:19088-94.

8. Aoyama T, Uchida Y, Kelley RI, Marble M, Hofman K, Tonsgard JH, et al. A novel disease with deficiency of mitochondrial very-long-chain acyl-CoA dehydrogenase. Biochem. Biophys Res Commun 1993; 191:1369-72.

9. Izai $K$, Uchida $Y$, Orii T, Yamamoto $S$, Hashimoto T. Novel fatty acid $\beta$-oxidation enzymes in rat liver mitochondria. I. Purification and properties of very-long chain acyl-coenzyme A dehydrogenase. J Biol Chem 1992; 267:1027-33.

10. Aoyama T, Souri M, Ushikubo S, Kamijo T, Yamaguchi S, Kelley RI, et al. Purification of human verylong-chainacyl- coenzyme A dehydrogenase and characterization of its deficiency in seven patients. J Clin Invest 1995; 95:2465-73.

11. Andresen BS, Bross P, Vianey-Saban C, Divry P, Zabot MT, Roe CR, et al. Cloning and characterization of human very-long-chain acyl-CoA dehydrogenase cDNA, chromosomal assignment of the gene and identification in four patients of nine different mutations within the VLCAD gene. Hum Molec Genet 1996; 5:461-72.

12. Aoyama T, Souri M, Ueno I, Kamijo T, YamagushI S, Rhead WJ, et al. Cloning of human very-longchain acylcoenzyme A dehydrogenase and molecular characterization of its deficiency in two patients. Am J Hum Gen 1995; 57:273-83.

13. Vianey-Saban C, Divry P, Brivet M, Nada M, Zabot MT, Mathieu M, et al. Mitochondrial very-long-chain acylcoenzyme $A$ dehydrogenase deficiency: clinical characteristics and diagnostic considerations in 30 patients. Clin Chim Acta 1998; 269:43-62.

14. Olgivie I, Pourfarzam M, Jackson S, Stockdale C, Bartlett K, Turnbull DM. Very long-chain acyl coenzyme A dehydrogenase deficiency presenting with exercise-induced myoglobinuria. Neurology 1994; 44:467-73.

15. Minetti C, Garavaglia B, Bado M, Invernizzi F, Bruno C, Rimoldi M, et al. Very-long-chain acyl-coenzyme A dehydrogenase deficiency in a child with recurrent myoglobinuria. Neuromuscul Disord 1998; 8:36.

16. Houten SM, Wanders RJ. A general introduction to the biochemistry of mitochondrial fatty acid B-oxidation. J Inherit Metab Dis 2010; 33(5):469-77. 
José Henry Osorio

17. Moczulski D, Majak I, Mamczur D. An overview of beta-oxidation disorders. Postepy Hig Med Dosw (Online) 2009; 63:266-77.

18. Kompare M, Rizzo WB. Mitochondrial fatty-acid oxidation disorders. Semin Pediatr Neurol 2008; 15(3):140-9.

19. Osorio JH, Pourfarzam M. Early diagnosis of neurometabolic diseases by tandem mass spectrometry. Acylcarnitine profile from cord blood. Rev. Neurol. 2004; 38(1):11-16.

20. Kølvraa s, Gregersen N, Christiensen E, Hobolth N. In vitro fibroblasts studies in a patient with C6C10 dicarboxilic aciduria: evidence for a defect in general acyl-CoA dehydrogenase. Clin Chim Acta $1982 ; 126: 53-67$.

21. Manning NJ, Olpin SE, Pollit RJ, Webley JA. Comparison of 9.10-3HPalmitic and 9.10-3Hmyristic acids for the detection of defects of fatty acid oxidation in intact cultured fibroblasts. J Inher Metab. Dis $1990 ; 13: 58-68$.

22. Olpin SE, Manning NJ, Carpenter K, Middleton B, Pollit RJ. Differential diagnosis of hydroxydicarboxylic aciduria based on release of $3 \mathrm{H} 2 \mathrm{O}$ from $[9,10-3 \mathrm{H}]$-myristic and $[9,10-3 \mathrm{H}]$-palmitic acids by intact cultured fibroblasts. J Inher Metab Dis 1992; 15:883-890.

23. Osorio $\mathrm{JH}$, Lluch M, Ribes A. Analysis of organic acids after incubation with (16-2H3)palmitic acid in fibroblasts from patients with mitochondrial beta-oxidation defects. J Inherit Metab Dis. 2003; 26(8):795-803. 